\title{
Managing Energy Efficiency in Manufacturing Processes - Implementing Energy Performance in Production Information Technology Systems
}

\author{
Katharina Bunse and Matthias Vodicka \\ ETH Zurich, BWI Center for Enterprise Sciences, 8092 Zurich, Switzerland \\ kbunse@ethz.ch, mvodicka@ethz.ch
}

\begin{abstract}
Climate change and unsecured energy supply are topics that become increasingly important in today's society. Although renewable energy technologies may be a long-term solution, more efficient energy use potentially makes a high and economic contribution to the given challenges in the short term. New $\mathrm{CO}_{2}$ regulations, rising energy prices and environmentally aware customers make energy efficient manufacturing a priority topic on the agenda of industrial companies. This paper focuses on drives for industrial companies to become more energy efficient in their manufacturing processes by integrating energy efficiency performance information and communication technology (ICT) in production management systems. Based on an EU roadmapping project, the paper analyzes which management tools and concepts support manufacturing companies to successfully implement energy efficiency improvement measures. By integrating energy efficiency performance criteria in ICT systems, decision-makers in industrial companies will be supplied with real-time and accurate information enabling them to make more effective business decisions.
\end{abstract}

Keywords: Energy Efficient Manufacturing, Information and Communication Technology, Production Management.

\section{Introduction}

Climate change and unsecured energy supply are topics that are becoming increasingly important in today's society and economy. Using the available energy more efficiently is one approach in meeting rising energy needs [1]. Furthermore, energy efficiency helps in preventing fossil fuel depletion, enhancing a nation's energy security, and preventing further deterioration of environmental quality [2]. With its share of $33 \%$ of final energy consumption, the manufacturing industry is one of the main energy consumers and the largest emitter of $\mathrm{CO}_{2}$ emissions [3]. Not only from a policy and society perspective does energy efficiency hold great benefits, there are also numerous advantages from an industrial perspective in becoming more energy efficient in manufacturing processes. There are three main reasons for companies to implement energy efficiency improvements: rising energy prices, new environmental regulations with their associated cost for emissions, and customers 
changing their purchasing behavior with regard to environmentally benign products and services. In the energy-intensive manufacturing industries (e.g., steel, cement, pulp and paper, chemicals), energy costs can represent up to $60 \%$ of operating costs [4].

Moreover, energy efficiency is an important pillar of sustainable business strategies. The move towards sustainability comes with many benefits for industrial companies. Willard [5] suggests seven major benefits from adopting a sustainability business strategy for industrial companies: (a) easier hiring of the best talent, (b) higher retention of top talent, (c) increasing employee productivity, (d) reduced expenses in manufacturing, (e) reduced expenses at commercial sites, (f) increased revenue/market share, and $(\mathrm{g})$ reduced risk and easier financing. Examples in literature and practice show that although the manufacturing sector has made continuous improvements in energy efficiency over the last years, "the economic energy efficiency potential in the industrial sector is far from being exhausted" [6]. Moreover, some researchers in the field of energy efficiency suggest that there are productivity benefits that can be associated with energy efficiency improvement measures [7, 8, 9]. These non-energy benefits could be, for example, lower maintenance costs, increased production yield, safer working conditions and many others. Worell et al. [10] argue that additional productivity benefits should be included in an economic assessment of the potential of energy efficiency improvements.

This paper focuses on the integration of energy efficiency performance criteria into production management systems as an enabler for companies to become more energy efficient in their manufacturing process. As IT infrastructure is increasing drastically since the 80's [11] appropriate ICT systems in manufacturing are significant drivers for successful businesses. Based on a EU funded roadmapping project this paper analyses which ICT related management approaches and concepts need to be adapted by companies in order to successfully implement energy efficiency improvement measures. In this paper we want to highlight the most important and promising research areas for utilizing ICT for energy efficient manufacturing.

\subsection{Initial Background and Problem Definition}

As mentioned before, there are several reasons and incentives for industrial companies to implement energy efficiency improvements in their manufacturing process. Nevertheless, several studies show that even profitable investments in energy efficiency are sometimes not undertaken by companies, and that there exists a gap between potential and actual energy saving measures (e.g., [6, 12, 13, 14.). The "energy efficiency gap" debate focuses on the reasons why profitable investments to reduce energy consumption are not realized in companies. One reason may be that in many companies, particularly small and medium-sized companies, investments except for buildings and infrastructure - are decided on according to payback periods instead of internal interest rate calculations [15]. Unrealistic high implicit discount rates in standard investment models are one of the reasons that profitable energy efficiency technologies are not implemented ([12, 15]). Moreover, energy investment costs include "difficult-to-measure components, such as transaction costs, monitoring costs, administrative costs, and adjustment costs" [16]. The most important barriers 
for investments in energy-saving technologies may be "other more attractive investment opportunities, incomplete depreciation of the existing capital stock" [13], and not important enough energy costs.

Some studies have also identified the low status of energy management as a barrier to energy efficiency [17]. The most important paradoxes and problems associated with the development and implementation of Total Quality Environmental Management (TQEM) systems are, among others: a strong bias in favor of ignorance at the highest management levels of the firms, a real concern as to whether customers are willing to pay the added costs associated with having something that is environmentally friendly, fear of negative returns, lack of appropriate measures and tools for capturing the environmental impact (see [18]).

In this paper we argue that ICT holds a high potential for companies to overcome some of the barriers responsible for the energy efficiency gap. By making information available and energy saving and financial savings from energy efficiency investments transparent, by reducing complexity, or by automatically controlling the production process with regard to energy efficiency, ICT is a major enabler for energy efficient manufacturing.

\subsection{Objectives of the Paper}

This paper demonstrates that integration of energy efficiency performance criteria in ICT systems is a highly relevant topic for manufacturing companies nowadays. The IT infrastructure of industrial companies consists of many different systems. Enterprise Resource Planning (ERP) Systems, Manufacturing Execution Systems (MES), Supply Chain Management systems (SCM), Customer Relationship Management (CRM), and Product Lifecycle Management (PLM) are important ICT tools and have a high impact on business processes [19]. Moreover, measurement and control systems are an integral part of the manufacturing system.

This paper highlights the need for future research in the area of ICT enabling energy efficiency in manufacturing processes. First, it demonstrates that this research area is highly relevant. Second, the paper gives more details on the concepts and tools necessary for integrating energy efficiency Key Performance Indicators (KPIs) into production management systems. It concludes with a discussion and outlook on future collaborative research activities.

\section{Methodology}

This research is based on the results of the EU funded project IMS2020, which has the objective of supporting future manufacturing environments by building a roadmap and highlighting the main milestones for future research activities needed to achieve a desired vision for manufacturing systems. IMS2020 is embedded in the global activities of the Intelligent Manufacturing Systems (IMS) initiative. IMS is a platform for global collaborative research and experience exchange. At the time of writing this paper Japan, Korea, Europe (EU incl. Norway, and Switzerland), and the United States are participating in the IMS initiative. IMS2020 focuses on five research areas, the so-called Key Area Topics (KAT), namely Sustainable Manufacturing, Energy 
Efficient Manufacturing, Key Technologies, Standards and Education. The paper at hand is based on the results of this project in the area of Energy Efficient Manufacturing (EEM). In order to identify the needs and challenges of industrial companies a thorough state-of-the-art analysis was conducted. In parallel, 106 interviews, an online survey with 261 participants, and several workshops were conducted in order to gather experience in the field of EEM and to gain up-to-date information about trends in this area. The identified topics were consolidated to 62 research topics and sent again to industrial and academic experts to evaluate their relevance in a second online survey with 356 participants. The development of the roadmaps has been supported by collaborative tools shared with all the Roadmapping Support Group, a growing community that, at the moment, counts 254 participants from 108 mainly industrial organizations.

\section{Integrating Energy Efficiency Performance Criteria into ICT of Production Management Systems}

In the area of EEM, 11 research topics were identified. They were clustered into four research areas called: "Energy Sources for Factories", "Efficient Production Processes", "Energy Utilization in Collaborative Frameworks" and "Management and Control of Energy Consumption" [20]. The second most important research topic within the Key Area Topic EEM with a mean relevance of 3.03 (where 1 means "very low relevance" and 4 means "very high relevance") was the research topic "Integrating Energy Efficiency Performance Criteria into ICT production management systems". Moreover, around $80 \%$ of the participants stated that they are interested in collaborative research regarding this topic, now or in the future.

In the interviews with industry representatives it became evident that most of the present production planning and control systems do not integrate energy efficiency as a relevant performance criterion. ICT plays an enabling role for energy efficiency improvements - either as a tool to help companies track their energy consumption and identify areas where savings can be made, or as the basis for more efficient production concepts and techniques. An enhanced framework for managing and optimizing energy efficiency in manufacturing processes needs to be developed and implemented in enterprise production information systems, as depicted in table 1, which shows some examples of industrial needs mentioned in the interviews.

Research should aim at extending the scope of MES, ERP, and SCM software to achieve energy efficiency goals. MES deliver information that enables the optimization of production processes from order receipt until outgoing goods [21]. As MES enable production managers and the process owner to understand how resources are used in the production process, they hold a significant potential for enhancing energy efficiency in production. Thus, a framework should be developed with the aim of showing how MES can be enhanced towards energy efficiency performance and how it can be implemented software-wise. A study from Rockwell Automation states: "As control systems and MESs increase the amount of information available to manufacturers, data management will become a more important part of ensuring that sustainability goals are met" [21]. Based on proper performance indicators and guiding management principles, MES are an important tool. For enhancing MES 
towards energy efficiency there are three important key technology areas: 1 . Sensor technology for monitoring and transmitting production asset performance, 2. Software for the evaluation of performance data, 3. Management concepts to derive appropriate improvement strategies.

Table 1. Examples for research areas where ICT acts as enabler of EEM in order to coordinate actions and increase the benefits of energy efficiency improvement activities (based on interviews with representatives of manufacturing companies)

\section{Examples for research areas where ICT acts as enabler of EEM (based on interviews)}

Plant-wide solutions for energy efficiency and process integration.

Full and dynamic integration of information from the shop-floor to top-floor (e.g. integration with MES).

Open Information Systems for small and medium sized companies (SMEs), enhancing functionalities of ERP/SCM/CRM software for an effective and efficient integration into whole Supply Chain.

Development of ubiquitous information platform which visualize manufacturing processes holistically.

Making key business processes more transparent and visible throughout the company's environment and include technologies as voice recognition, RFID tagging.

PLM systems to manage dynamically and in real-time all necessary information in order to make better and quicker management decisions

Standardization of data down broken to the shop-floor may result in a new generation of inter-organizational platforms for the exchange of data between ERP-Systems.

MES typically have interfaces to the automation and control systems and to ERP systems [21]. Modern ERP systems incorporate the resource planning and business processes of the entire company. Improving the shop floor scheduling and planning of the EPR system contributes to increased energy efficiency. ERP systems often have an interface to SCM modules for providing data for network optimization. The optimization that is performed by these software tools should also take into consideration the implications in terms of energy efficiency. Fig. 1 highlights important elements to be considered, when enhancing ICT production management systems towards energy efficiency.

By setting up production information systems towards EEM, decision makers will be provided with relevant information about impacts on energy performances due to production planning and business decisions. Hence more energy-aware and effective decisions can be taken, improving the enterprise performances in terms of energy efficiency. Individual IT-solutions can play a central role for increasing energy efficiency in many sectors [23]. Virtualization tools and digital manufacturing engineering require further improvement in order to assist organizations in reducing their expenditures for energy and emissions ([24, 25]). Information technology has the 
potential to help manage and control the energy consumption in the industrial and public sector. A study by McKinsey reveals that the global market potential for energy management-IT-solutions will be around 15 billion EUR in the year 2020 . This correlates with an annual growth of 14\% from the year 2008 [23]. The European Commission also highlighted the relevance of ICT for energy efficiency in manufacturing, especially focusing on the potentials of intelligent controls, sensor and actuator networks, smart components and systems, asset monitoring systems, and advanced scheduling algorithms [26].

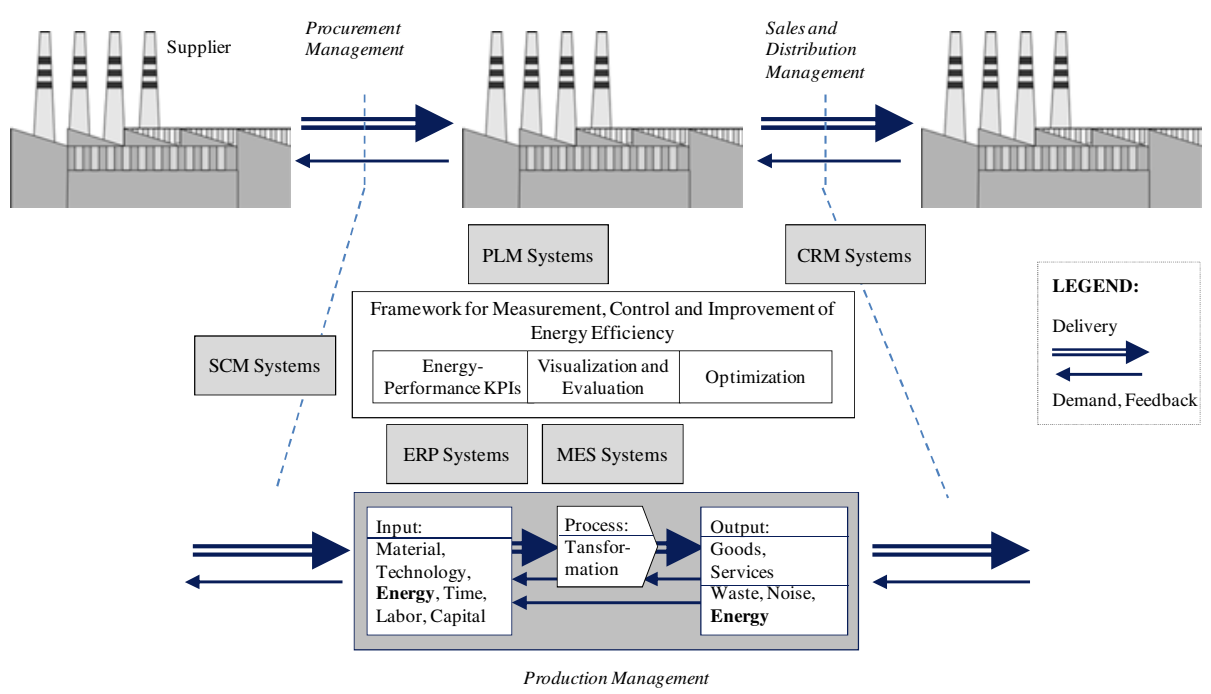

Fig. 1. Important elements for ICT enabling energy efficient manufacturing (adapted from [22])

A report by the climate group on behalf of the Global eSustainability Initiative (GeSI) states that the ICT sector can be expected to be responsible for $1.43 \mathrm{GtCO}_{2}$ emissions in the year 2020 (about $3 \%$ of global $\mathrm{CO}_{2}$ emissions in a business-as-usual scenario), while ICT as an enabler can also lead to $\mathrm{CO} 2$ emission reduction of about $7.8 \mathrm{GtCO}_{2}$ emissions by 2020 [27]. For achieving this reduction in energy consumption and $\mathrm{CO}_{2}$ emissions ICT driven manufacturing systems and optimization in logistics networks will be critical factors. The development of EEM oriented IT solutions requires the integration of various production levels - from shop floor to management level. Moreover, the definition of suitable performance indicators is a basic requirement for an ICT enabled management of energy efficiency. In [28] a selection of energy efficiency KPIs can be found, which are implemented in manufacturing companies, especially in the energy-intensive industries. Two typical indicators are the energy intensity (EI) and the specific energy consumption (SEC). EI is called an economic indicator as it is derived from economic terms. In contrast, the SEC relates to physical units per tonne of product and is thus called a physical indicator.

When integrating energy performance in ICT systems for production management, a special attention has to be given to the interface between the different systems. MES 
and ERP systems, for example, should be linked in order to integrate the financial perspective and the dynamics of the shop floor (e.g., machine utilization and downtime). Lee suggests that this link "is hindered by the lack of integrated information coming from and flowing to control systems on the plant floor" [19]. There are also limitations of ERP systems to be utilized for SCM planning and optimization. Current ERP are not sufficient in their modularity, openness and flexibility for supporting SCM across multiple enterprises [29]. Besides the software and tool development, education and training of employees and an enterprise culture with the flexibility of local dynamic decision-making are critical factors for the successful implementation of new production management ICT solutions and tools [19].

In the discussion about enhancing ICT systems towards supporting EEM special attention has to be given to the fact, that ICT systems are not only enablers for energy efficiency but are also main consumers of energy. ICT systems are responsible for the same amount of $\mathrm{CO}_{2}$ emissions as global air travel due to the increase in power required for rising data rates and growing market penetration of ICT solutions [30]. Moreover, due to the rebound effect - that refers to an effective increase of energy consumption as a consequence of increasing energy efficiency and the associated decrease in energy price - the overall effect of increasing energy efficiency through enhanced ICT systems is very difficult to assess [31]. Hilty et al. [32] identified positive and negative environmental impacts from ICT on different levels (first, second and third order effects). Yi and Thomas [33] reviewed existing literature and research projects on the environmental impact of ICT and concluded "that traditional assessment approaches are insufficient to accommodate the digital technology revolution and cannot accommodate the challenge of measuring the impacts of ICT on environmental sustainability" [33].

\section{Discussion and Conclusion}

Adapted ICT systems for energy efficiency may enhance a company's IT infrastructure to support strategies striving for sustainable business activities. By making relevant production and process data transparent and available to decisionmakers in real time, energy efficiency improvements would be supported in various ways: On the one hand, benefits and financial savings from energy efficiency improvements may gain visibility, mitigating barriers to energy efficiency improvements. On the other hand, ERP and MES systems allow for more energy efficient production processes by specifically controlling the processes with regard to energy efficiency. However, to accomplish latter suitable energy efficiency, KPIs have to be defined and integrated into these systems. In this area also standardization activities are required to allow for benchmarks. Further, new interfaces for solutions from different providers need to be established. Concluding, we suggest, that future research should aim at enablers, such as concepts and tools, supporting the integration of energy efficiency performance criteria into ICT systems for production management.

In order to develop new energy management systems, the sensors and control devices require attention as well as the KPIs and the techno-human interfaces. With 
this, energy efficiency can become an integral part of the manufacturing systems and can be represented in the ICT systems. In future research projects, enhanced frameworks for managing and optimizing energy efficiency need to be developed and implemented in enterprise planning and control information systems. Furthermore, investigation on the overall effect of enhancing ICT with regard to energy efficiency performance indicators, especially taking into consideration the rebound effect, may be undertaken.

\section{References}

1. International Energy Agency (IEA): Assessing measures of energy efficiency performance and their application in industry, http: / /www. iea.org

2. Mukherjee, K.: Energy use efficiency in U.S. manufacturing: A nonparametric analysis. Energy Economics 30, 76-96 (2008)

3. International Energy Agency (IEA): Worldwide Trends in Energy Use and Efficiency, Key Insights from IEA Indicator Analysis, http: / /www. iea. org

4. International Energy Agency (IEA): Feedstock Substitutes, Energy Efficient Technology and CO2 Reduction for Petrochemical Products. In: Workshop Proceedings,

http: //www. iea.org

5. Willard, B.: The sustainability advantage: Seven business case benefits of a triple bottom line. New Society Publ., Gabriola Island, B.C. (2002)

6. Eichhammer, W.: Industrial Energy Efficiency. In: Cleveland, C.J. (ed.) Encyclopedia of Energy, pp. 383-393. Elsevier, New York (2004)

7. Elliott, R.N., Latiner, S., Pye, M.: Considerations in the estimation of cost and benefits of industrial energy efficiency projects. In: Cairns, E.J. (ed.) Proceedings of the ThirtySecond Intersociety Energy Conversion Engineering Conference. American Institute of Chemical Engineers, New York (1997)

8. Porter, M.E., van der Linde, C.: Green and Competitive: Ending the Stalemate. In: Harvard Business Review, pp. 120-134 (September-October 1995)

9. Boyd, G.A., Pang, J.X.: Estimating the linkage between energy efficiency and productivity. Energy Policy 28, 289-296 (2000)

10. Worrell, E., Laitner, J.A., Ruth, M., Finman, H.: Productivity benefits of industrial energy efficiency measures. Energy 28, 1081-1098 (2003)

11. Krcmar, H.: Informationsmanagement. Springer, Berlin (1997)

12. DeCanio, S.J.: The efficiency paradox: bureaucratic and organizational barriers to profitable energy-saving investments. Energy Policy 26, 441-454 (1998)

13. De Groot, H.L.F., Verhoef, E.T., Nijkamp, P.: Energy saving by firms: decision-making, barriers and policies. Energy Economics 23, 717-740 (2001)

14. Paton, B.: Efficiency gains within firms under voluntary environmental initiatives. Journal of Cleaner Production 9, 167-178 (2001)

15. Anderson, S.T., Newell, R.G.: Information programs for technology adoption: the case of energy-efficiency audits. Resource and Energy Economics 26, 27-50 (2004)

16. DeCanio, S.J., Watkins, W.E.: Investment in Energy Efficiency: Do the Characteristics of Firms Matter? The Review of Economics and Statistics 80, 95-107 (1998)

17. SPRU (Science and Technology Policy Research): Reducing barriers to energy efficiency in public and private organizations Brighton, UK (2000) 
18. Curkovic, S., Sroufe, R.: Total Quality Environmental Management and Total Cost Assessment: An exploratory study. Scheduling in batch-processing industries and supply chains. International Journal of Production Economics 105, 560-579 (2007)

19. Lee, J.: E-manufacturing - fundamental, tools, and transformation. Leadership of the Future in Manufacturing. Robotics and Computer-Integrated Manufacturing 19, 501-507 (2003)

20. IMS2020. Action Roadmap on Key Areas 1, 2 and 3, http://data.fir.de/projektseiten/ims2020/files/ Action_Roadmap.pdf

21. Rockwell Automation: Manufacturing Execution Systems for Sustainability. Extending the Scope of MES to Achieve Energy Efficiency and Sustainability Goals, http: / / literature.rockwellautomation.com

22. Schönsleben, P.: Integral logistics management. Operations and Supply Chain Management in Comprehensive Value-Ndded Networks. Auerbach Publ., Boca Raton (2007)

23. McKinsey Deutschland: Wettbewerbsfaktor Energie. Neue Chancen für die deutsche Wirtschaft, http: //www. mckinsey.de

24. European Commission (EC): The Future of Manufacturing in Europe 2015-2020 - The Challenge for Sustainability - Scenarios, http://simula.cesga.es/document/ docs/TheFuturemanufacturing.pdf

25. European Commission (EC): Manufuture - Strategic Research Agenda - Report of High Level Group, http: / / manufuture.org

26. European Commission (EC): ICT and energy efficiency - the case for manufacturing. Recommendations of the Consultation Group, http : / ec . europa . eu

27. The Climate Group: SMART 2020: Enabling the low carbon economy in the information age, http: / / www. smart2020.org

28. Bunse, K., Sachs, J., Vodicka, M.: Evaluating Energy Efficiency Improvements in Manufacturing Processes. In: Proceedings of the International Conference on Advances in Production Management Systems Conference, Bordeaux, France (2009)

29. Akkermans, H.A., Bogerd, P., Yücesan, E., van Wassenhove, L.N.: The impact of ERP on supply chain management: Exploratory findings from a European Delphi study. European Journal of Operational Research 146, 284-301 (2003)

30. Fettweis, G., Zimmermann, E.: ICT Energy Consumption - Trends and Challenges. In: The 11th International Symposium on Wireless Personal Multimedia Communications (2008)

31. Plepys, A.: The grey side of ICT. Sustainability in the Information Society. Environmental Impact Assessment Review 22, 509-523 (2002)

32. Hilty, L.M., Arnfalk, P., Erdmann, L., Goodman, J., Lehmann, M., Wäger, P.A.: The relevance of information and communication technologies for environmental sustainability - A prospective simulation study. Environmental Informatics. Environmental Modelling and Software 21, 1618-1629 (2006)

33. Yi, L., Thomas, H.R.: A review of research on the environmental impact of e-business and ICT. Environment International 33, 841-849 (2007) 